\title{
A limitation of the estimation of intrinsic volumes via pixel configuration counts*
}

\author{
Jürgen Kampf, \\ TU Kaiserslautern, postbox 3049, 67653 Kaiserslautern, Germany, \\ phone: 0049631205 4825, fax: 0049631205 2748, kampf@mathematik.uni-kl.de
}

September 28, 2012

\begin{abstract}
It is often helpful to compute the intrinsic volumes of a set of which only a pixel image is observed. A computational efficient approach, which is suggested by several authors and used in practice, is to approximate the intrinsic volumes by a linear functional of the pixel configuration histogram. Here we want to examine, whether there is an optimal way of choosing this linear functional, where we will use a quite natural optimality criterion that has already been applied successfully for the estimation of the surface area. We will see that for intrinsic volumes other than volume or surface area this optimality criterion cannot be used, since estimators which ignore the data and return constant values are optimal w.r.t. this criterion. This shows that one has to be very careful, when intrinsic volumes are approximated by a linear functional of the pixel configuration histogram.
\end{abstract}

Keywords: Intrinsic volume; Pixel image; Integral of mean curvature; Euler characteristic; Optimal estimation

\section{Introduction}

Intrinsic volumes are a fundamental concept of convex and integral geometry with many applications, e.g. in the analysis of microstructures. We denote the intrinsic volumes of a set $K \subseteq \mathbb{R}^{d}$ from the convex ring by $V_{k}(K), k=0, \ldots, d$; see [9, sections $4.1,4.2$ and 4.4] for a detailed introduction or [10, section 14.2] for a shorter introduction. In particular, $V_{d}(K)$ equals the volume of $K, V_{d-1}(K)$ is half the surface area of $K$, and $V_{0}(K)$ is the Euler-characteristic of $K$. The Euler-characteristic is an integer-valued signed topological quantity that plays an important role in several branches in geometry and topology. If $d=2$, then $V_{0}(K)$ equals the difference between the number of connected components of $K$ and the number of bounded connected components of $\mathbb{R}^{2} \backslash K$. If $K$ is a 1-dimensional set in $\mathbb{R}^{3}$, e.g. the edge system of a mosaic, then $V_{1}(K)$ is the length of $K$.

In applications often the problem arises to compute intrinsic volumes of a set (at least approximately), even though only a pixel image of this set is observed. In this paper we always assume that the pixels are the points of the lattice $t \mathbb{Z}^{d}, t>0$, and that a pixel is colored black if it lies in the set and it is colored white if it does not lie inside the set, i.e. the information contained in the image is equivalent to the information contained in the Gauss digitization of the observed set. Several algorithms have been proposed for the computation of arbitrary intrinsic volumes (see [6] and the literature cited in there) and even more algorithms exist for the surface area (see [5] and the literature cited in there) and the Euler characteristic (see [2] and the literature cited in there). A computationally efficient approach, that was proposed by several authors - e.g. by Gray [1] for the Euler characteristic in the plane and by Ohser, Nagel and Schladitz [7] for arbitrary intrinsic volumes - and that is used in practice, is to approximate the intrinsic volume by a linear functional of the pixel configuration histogram. A $2^{d}$-pixel configuration

*This work was supported by the German Federal Ministry for Education and Research (BMBF) under grant 03MS603A. 
is a pair $(B, W)$ of two disjoint sets whose union is $\{0,1\}^{d}$. The interpretation is that $B$ is the set of black (foreground) pixels and $W$ is the set of white (background) pixels. We enumerate the $2^{d}$-pixel configuration as $\left(B_{i}, W_{i}\right), i=1, \ldots, 2^{\left(2^{d}\right)}$. For a set $K \subseteq \mathbb{R}^{d}$, a number $i \in\left\{1, \ldots, 2^{\left(2^{d}\right)}\right\}$ and lattice distance $t>0$ we let $N_{t, i}(K)$ denote the number of occurrences of configuration $i$ in an image of $K$ in the observation window $[-1 ; 1]^{d}$. More precisely, we put

$$
N_{t, i}(K):=\#\left\{z \in t \mathbb{Z}^{d} \cap[-1 ; 1-t]^{d} \mid z+t B_{i} \subseteq K, z+t W_{i} \subseteq K^{C}\right\},
$$

where $K^{C}$ denotes the complement of the set $K$ and \#S denotes the number of elements of a set $S$ (observe that a $2^{d}$-pixel configuration is contained in $[-1 ; 1]^{d}$ iff its "lower left" corner is in $[-1 ; 1-t]^{d}$ ). Now we consider two pixel configurations to be equivalent if there is an isometry mapping one of them to the other. We let $\nu_{d}$ be the number of equivalence classes of $2^{d}$-pixel configurations and enumerate the equivalence classes by $0, \ldots, \nu_{d}-1$. We let $\tilde{N}_{t, j}(K)$ denote the sum over all $N_{t, i}(K)$ such that the configuration $i$ belongs to equivalence class $j$.

Let $\mathbb{R}^{+}$denote the set of positive real numbers. As mentioned above, linear functionals of the pixel configuration histogram, i.e. functionals that map $K \subseteq \mathbb{R}^{d}$ to

$$
\sum_{j=0}^{\nu_{d}-1} \lambda_{j}(t) \tilde{N}_{t, j}(K),
$$

where the functions $\lambda_{0}, \ldots, \lambda_{\nu_{d}-1}: \mathbb{R}^{+} \rightarrow \mathbb{R}$ are chosen properly and called weights, can be used as computational efficient estimators for $V_{k}(K)$. A geometrically motivated proposal for the weights was made in [7]. This leads to the question, whether there is an optimal way of choosing these weights. An optimality criterion was proposed and successfully applied in the special case $k=d-1$ by Kiderlen and Ziegel [4]. They assumed that the set $K$ is shifted at random and then considered the worst case asymptotic relative bias of the estimator $\hat{V}_{d-1}$, that is

$$
\sup _{K} \limsup _{t \rightarrow 0} \frac{\left|\mathbb{E} \hat{V}_{d-1}(K+t U)-V_{d-1}(K)\right|}{V_{d-1}(K)},
$$

where $U$ is a random vector distributed uniformly on $[0 ; 1]^{d}$ and the supremum is taken over all sets $K$ for which the estimator is supposed to work.

Since the $k$-th support measure $\Xi_{k}$ (for an introduction see e.g. [10, section 14.2]), whose total mass the $k$-th intrinsic volume is, is a signed measure for $k<d-1$, extinction effects may occur and hence it does not seem to be a good idea to measure the bias of an estimator for $V_{k}$ relative to the correct value (unless we choose a very small class of sets on which our estimator should work). A second idea may be to measure the bias of the estimator for $V_{k}$ always relative to $V_{d-1}$. However, if one considers $\mu K$ for a fixed set $K$, then the quotient of the bias of $\hat{V}_{k}$ and $V_{d-1}$ is proportional to $\mu^{k+1-d}$ (if $\hat{V}_{k}$ is homogenous of degree $k$ ) and hence gets arbitrarily large for $\mu \rightarrow 0$ if $k<d-1$. The solution we will use is based on the following idea: Heuristically spoken, the bias of $\hat{V}_{k}$ is the sum of all errors made in the estimation of positive part and all errors made in the estimation of the negative part of $\Xi_{k}$. Hence it seems to be a good idea to measure the bias of $\hat{V}_{k}$ relative to the total variation of $\Xi_{k}$, which is not zero by Lemma 8 below. We denote the total variation of $\Xi_{k}, k=0, \ldots, d-1$, by $\left|V_{k}\right|$ and put for completeness $\left|V_{d}\right|:=V_{d}$. While Kiderlen and Ziegel managed to find weights for which the worst case asymptotic relative bias of the estimator for $V_{2}$ in $\mathbb{R}^{3}$ is $4 \%$, we will show that there are no weights for which the relative worst case asymptotic bias of the estimator for $V_{0}, \ldots, V_{d-2}$ is below $100 \%$. Since an estimator that ignores the data and returns 0 never has a relative bias of more than $100 \%$, such a useless estimator has the smallest worst case asymptotic relative bias among all estimators of type (1). This is already true on a quite small class of sets, namely on

$$
\mathcal{P}^{(2)}:=\left\{\bigcup_{i=1}^{N} P_{i} \mid P_{i} \text { is (convex) polytope in } \mathbb{R}^{2} \text { with interior points, } i=1, \ldots, N, N \in \mathbb{N}\right\} .
$$

resp. on the class

$$
\begin{aligned}
\mathcal{P}^{(d)}:= & \left\{P \times\left[a_{1}, b_{1}\right] \times \cdots \times\left[a_{d-2}, b_{d-2}\right] \mid\right. \\
& \left.P \in \mathcal{P}^{(2)}, a_{1}, \ldots, a_{d-2} \in \mathbb{R}, b_{1}, \ldots, b_{d-2} \in \mathbb{R}, a_{1}<b_{1}, \ldots, a_{d-2}<b_{d-2}\right\}, \quad d \geq 3 .
\end{aligned}
$$


Now we are in the position to give the precise formulations of the main results of the present paper.

Theorem 1. For all weights $\lambda_{0}, \ldots, \lambda_{\nu_{d}-1}$ and all $n \in \mathbb{N}, n \geq 2$, the estimator $\hat{V}_{0}$ for $V_{0}$ defined by (1) has a worst case asymptotic bias of at least $n-1$ on the set

$$
\left\{K \in \mathcal{P}^{(d)}|| V_{0} \mid(K) \leq n, K \subseteq(-1 ; 1)^{d}\right\} .
$$

The estimator ignoring the data and returning 1 has a worst case (asymptotic) bias of $n-1$. Hence this estimator has the minimal worst case asymptotic bias among all estimators of type (1).

The proof of Theorem 1 will be given in Section 4. First it will be established in the 2-dimensional case, then it will be extended to the higher-dimensional case using cylinder sets.

The following corollary is a direct consequence of Theorem 1.

Corollary 2. For all weights $\lambda_{0}, \ldots, \lambda_{\nu_{d}-1}$ the estimator $\hat{V}_{0}$ for $V_{0}$ defined by (1) has a worst case asymptotic relative bias of at least $100 \%$ on the set

$$
\left\{K \in \mathcal{P}^{(d)} \mid K \subseteq(-1 ; 1)^{d}\right\} .
$$

The estimators ignoring the data and returning 0 or 1 have a worst case (asymptotic) relative bias of $100 \%$. Hence these estimators have the minimal worst case asymptotic relative bias among all estimators of type (1).

The following theorem is an extension of Theorem 1 to other intrinsic volumes. The extension from Theorem 1 to Theorem 3 uses cylinder sets with a $(d-k)$-dimensional base and will be given in Section 4 .

Theorem 3. For all weights $\lambda_{0}, \ldots, \lambda_{\nu_{d}-1}$, all $k=1, \ldots, d-2$ and all real numbers $r \geq 2\left(\begin{array}{l}d \\ k\end{array}\right)$, the estimator $\hat{V}_{k}$ for $V_{k}$ defined by (1) has a worst case asymptotic bias of at least $r$ on the set

$$
\left\{K \in \mathcal{P}^{(d)}|| V_{k} \mid(K) \leq r, K \subseteq(-1 ; 1)^{d}\right\} .
$$

The estimator ignoring the data and returning 0 has a worst case (asymptotic) bias of $r$. Hence this estimator has the minimal worst case asymptotic bias among all estimators of type (1).

As an immediate consequence of Theorem 3 we obtain the following corollary.

Corollary 4. For all weights $\lambda_{0}, \ldots, \lambda_{\nu_{d}-1}$ and all $k=1, \ldots, d-2$ the estimator $\hat{V}_{k}$ for $V_{k}$ defined by (1) has a worst case asymptotic relative bias of at least $100 \%$ on the set

$$
\left\{K \in \mathcal{P}^{(d)} \mid K \subseteq(-1 ; 1)^{d}\right\} .
$$

The estimator ignoring the data and returning 0 has a worst case (asymptotic) relative bias of $100 \%$. Hence this estimator has the minimal worst case asymptotic relative bias among all estimators of type (1).

These results have two consequences. First they show that the optimality criterion of having the least worst case asymptotic (relative) bias yields nonsense in the situation of these theorems. Second they tell us to be quite careful, when intrinsic volumes other than volume or surface area are estimated by a linear functional of the pixel configuration histogram, since such a estimators cannot be better than an estimator ignoring the data w.r.t. a quite reasonable optimality criterion.

However, there is still some hope for the estimation of intrinsic volumes from the pixel configuration histogram. In fact, Ohser, Nagel and Schladitz [7] have shown that even a diverging estimator may have a reasonable small error for the lattice distances used in practice.

Another possible solution to this limitation is to restrict to certain models. Anne-Marie Svane is preparing an article, where she determines the weights for which the estimator of the density of the Euler characteristic in $\mathbb{R}^{2}$ is asymptotically unbiased, when applied to a Boolean model. Moreover, she determines weights for which the estimator of the Euler characteristic in $\mathbb{R}^{2}$ is asymptotically unbiased, when applied 
to an isotropic random compact set fulfilling certain smoothness assumptions. Ohser et al. [8] examine weights such that the estimator for $V_{1}$ in $\mathbb{R}^{3}$ is unbiased for all sufficiently small lattice distances, when applied to an isotropic system of non-overlapping fibers.

This paper is organized as follows: In Section 2 we collect lemmata concerning intrinsic volumes and support measures and in Section 3 we deal with pixel configuration counts. Lemmata combining both topics will be given together with the proofs of the main results in Section 4.

\section{Intrinsic volumes and support measures}

A compact, non-empty subset of $\mathbb{R}^{d}$ will be called body. We let $\mathcal{N}(K)$ denote the normal bundle of a convex body $K \subseteq \mathbb{R}^{d}$, bd $A$ the boundary of a set $A \subseteq \mathbb{R}^{d}$ and int $A$ its interior.

Lemma 5. Let $K \subseteq \mathbb{R}^{d}$ be from the convex ring. Then

$$
\Xi_{0}(K, \mathcal{N}(\operatorname{conv} K))=1
$$

Proof. Using the index function approach for the extension of the support measures to the convex ring (see e.g. [9, p. 219-222]), it is easy to see that for all Borel sets $\eta \subseteq \mathcal{N}(\operatorname{conv} K) \cap\left((\right.$ bd $\left.K) \times S^{d-1}\right)$ we have

$$
\Xi_{k}(K, \eta)=\Xi_{k}(\operatorname{conv} K, \eta), \quad k=0, \ldots, d-1 .
$$

As a consequence of $[9,(4.6 .1)], \Xi_{0}(\operatorname{conv} K, \cdot)$ is concentrated on $($ bd $K) \times S^{d-1}$. Thus

$$
\Xi_{0}(K, \mathcal{N}(\operatorname{conv} K))=\Xi_{0}\left(\operatorname{conv} K, \mathcal{N}(\operatorname{conv} K) \cap\left((\operatorname{bd} K) \times S^{d-1}\right)\right)=V_{0}(\operatorname{conv} K)=1 .
$$

The fact that support measures are defined locally, which is well-known for convex bodies, is also true for sets from the convex ring:

Lemma 6. Let $K, L \subseteq \mathbb{R}^{d}$ be two sets from the convex ring and let $\eta \subseteq \mathbb{R}^{d} \times S^{d-1}$ be a Borel set. If there is an open set $O \subseteq \mathbb{R}^{d}$ with $K \cap O=L \cap O$ and $\eta \subseteq O \times S^{d-1}$, then

$$
\Xi_{k}(K, \eta)=\Xi_{k}(L, \eta), \quad k=0, \ldots, d-1 .
$$

Proof. Under the additional assumption that there are some $\epsilon>0$ and some open set $O^{\prime} \subseteq \mathbb{R}^{d}$ with $O^{\prime}+\epsilon B^{d} \subseteq O$ and $\eta \subseteq O^{\prime} \times S^{d-1}$, the statement is easily established using index functions. By standard theorems from measure theory, the result extends to the general case.

The following lemma is easily established using the formula for support measures of polytopes and Lemma 6.

Lemma 7. Let $L \subseteq \mathbb{R}^{d}$ be a set from the convex ring, such that there is a convex body $K \subseteq \mathbb{R}^{d}$ and pairwise disjoint convex bodies $K_{1}, \ldots, K_{n} \subseteq \operatorname{int} K$ with interior points such that $L=K \backslash \bigcup_{i=1}^{n}$ int $K_{i}$. Then $K_{1}, \ldots, K_{n}$ are polytopes and

$$
\Xi_{k}(L, \eta)=\Xi_{k}(K, \eta)+(-1)^{d-k+1} \sum_{i=1}^{n} \Xi_{k}\left(K_{i}, \eta^{*}\right)
$$

for $k=0, \ldots, d-1$, where $\eta^{*}=\{(x,-u) \mid(x, u) \in \eta\}$.

The following lemma is a bit unsatisfactory, since it is probably true for all sets $K \subseteq \mathbb{R}^{d}$ that can be represented as finite unions of convex bodies with interior points. However, it is not obvious how to prove this more general statement.

Lemma 8. Let $K \in \mathcal{P}^{(d)}, d \geq 2$, and $k \in\{0, \ldots, d-1\}$. Then $\Xi_{k}(K, \cdot)$ is not the zero measure. 
Proof. In the case $d=2, k=1$, this statement is obvious and, in the case $d=2, k=0$, it follows from Lemma 5. In the case $d \geq 3$, it follows by an induction w.r.t. $d$ from Lemma 9 below.

We denote by $e_{1}, \ldots, e_{d}$ the standard basis in $\mathbb{R}^{d}$, by $[x, y]$ the line segment with endpoints $x$ and $y$ and by $\kappa_{k}, k \in \mathbb{N}$, the volume of the $k$-dimensional unit ball. By $V_{d}$ we denote the Lebesgue measure. Since the Lebesgue measure coincides with the $d$-th intrinsic volume, whenever both notions are defined, the use of the same symbol is no problem.

Now we will show how the support measures of a cylinder set can be computed from the support measures of its base.

Lemma 9. Let $K \subseteq \mathbb{R}^{d-1}$ be from the convex ring and $h \geq 0$. For any Borel set $\eta \subseteq \mathbb{R}^{d} \times S^{d-1}$ we have

$$
\begin{aligned}
\Xi_{d-1}(K \times[0 ; h], \eta)= & \frac{1}{2} V_{d-1}\left(\left\{x \in K \mid\left((x, 0),-e_{d}\right) \in \eta\right\}\right) \\
& +\int_{0}^{h} \int_{\mathbb{R}^{d-1} \times S^{d-2}} \mathbf{1}_{\eta}((x, y),(u, 0)) \Xi_{d-2}(K, d(x, u)) d y \\
& +\frac{1}{2} V_{d-1}\left(\left\{x \in K \mid\left((x, h), e_{d}\right) \in \eta\right\}\right)
\end{aligned}
$$

and

$$
\begin{aligned}
\Xi_{k}(K \times[0 ; h], \eta) \\
=\frac{\kappa_{d-k-1}}{\kappa_{d-k}} \frac{d-k-1}{d-k} \int_{\mathbb{R}^{d-1} \times S^{d-2}} \int_{0}^{\pi / 2} \cos ^{d-k-2} \phi \mathbf{1}_{\eta}((x, 0),(\cos \phi \cdot u,-\sin \phi)) d \phi \Xi_{k}(K, d(x, u)) \\
\quad+\int_{0}^{h} \int_{\mathbb{R}^{d-1} \times S^{d-2}} \mathbf{1}_{\eta}((x, y),(u, 0)) \Xi_{k-1}(K, d(x, u)) d y \\
\quad+\frac{\kappa_{d-k-1}}{\kappa_{d-k}} \frac{d-k-1}{d-k} \int_{\mathbb{R}^{d-1} \times S^{d-2}} \int_{0}^{\pi / 2} \cos ^{d-k-2} \phi \mathbf{1}_{\eta}((x, h),(\cos \phi \cdot u, \sin \phi)) d \phi \Xi_{k}(K, d(x, u)), \\
\quad k=1, \ldots, d-2,
\end{aligned}
$$

and

$$
\begin{aligned}
\Xi_{0}(K \times[0 ; h], \eta) & \\
= & \frac{\kappa_{d-1}}{\kappa_{d}} \frac{d-1}{d} \int_{\mathbb{R}^{d-1} \times S^{d-2}} \int_{0}^{\pi / 2} \cos ^{d-2} \phi \mathbf{1}_{\eta}((x, 0),(\cos \phi \cdot u,-\sin \phi)) d \phi \Xi_{0}(K, d(x, u)) \\
& +\frac{\kappa_{d-1}}{\kappa_{d}} \frac{d-1}{d} \int_{\mathbb{R}^{d-1} \times S^{d-2}} \int_{0}^{\pi / 2} \cos ^{d-2} \phi \mathbf{1}_{\eta}((x, h),(\cos \phi \cdot u, \sin \phi)) d \phi \Xi_{0}(K, d(x, u)) .
\end{aligned}
$$

Proof. Assume additionally that $K$ is convex. For a convex body $L$ and $v \in \mathbb{R}^{d} \backslash L$ let $p(L, v)$ denote the metric projection of $v$ to $L$ and set $u(L, v):=(v-p(L, v)) /\|v-p(L, v)\|$.

Then for $r \geq 0$

$$
\begin{aligned}
& \sum_{k=0}^{d-1} \kappa_{d-k} r^{d-k} \Xi_{k}(K \times[0 ; h], \eta) \\
& \quad=V_{d}\left(\left\{v \in\left(K \times[0 ; h]+r B^{d}\right) \backslash(K \times[0 ; h]) \mid(p(K \times[0 ; h], v), u(K \times[0 ; h], v)) \in \eta\right\}\right) \\
& \quad=\int_{-r}^{r+h} V_{d-1}\left(\left\{x \in \mathbb{R}^{d-1} \mid(x, y) \in\left(K \times[0 ; h]+r B^{d}\right) \backslash(K \times[0 ; h]),\right.\right. \\
& \quad(p(K \times[0 ; h],(x, y)), u(K \times[0 ; h],(x, y))) \in \eta\}) d y
\end{aligned}
$$




$$
\begin{aligned}
=\int_{-r}^{0} & V_{d-1}\left(\left\{x \in K \mid\left((x, 0),-e_{d}\right) \in \eta\right\}\right) \\
& +V_{d-1}\left(\left\{x \in\left(K+\sqrt{r^{2}-y^{2}} B^{d-1}\right) \backslash K \mid\left((p(K, x), 0), \frac{(x-p(K, x), y)}{\|(x-p(K, x), y)\|}\right) \in \eta\right\}\right) d y \\
& +\int_{0}^{h} V_{d-1}\left(\left\{x \in\left(K+r B^{d-1}\right) \backslash K \mid((p(K, x), y),(u(K, x), 0)) \in \eta\right\}\right) d y \\
& +\int_{0}^{r} V_{d-1}\left(\left\{x \in K \mid\left((x, h), e_{d}\right) \in \eta\right\}\right) \\
& +V_{d-1}\left(\left\{x \in\left(K+\sqrt{r^{2}-y^{2}} B^{d-1}\right) \backslash K \mid\left((p(K, x), h), \frac{(x-p(K, x), y)}{\|(x-p(K, x), y)\|}\right) \in \eta\right\}\right) d y .
\end{aligned}
$$

We get

$$
\begin{aligned}
\int_{-r}^{0} V_{d-1}\left(\left\{x \in\left(K+\sqrt{r^{2}-y^{2}} B^{d-1}\right) \backslash K \mid\left((p(K, x), 0), \frac{(x-p(K, x), y)}{\|(x-p(K, x), y)\|}\right) \in \eta\right\}\right) d y \\
=\int_{-r}^{0} \sum_{k=0}^{d-2} \kappa_{d-k-1}(d-k-1) \int_{\mathbb{R}^{d-1} \times S^{d-2}} \int_{0}^{\sqrt{r^{2}-y^{2}}} s^{d-k-2} \\
=\sum_{k=0}^{d-2} \kappa_{d-k-1}(d-k-1) \int_{\mathbb{R}^{d-1} \times S^{d-2}} \int_{0}^{r} \int_{0}^{\pi / 2}(z \cdot \cos \phi)^{d-k-2} \\
=\sum_{k=0}^{d-2} \kappa_{d-k-1} \frac{d-k-1}{d-k} \int_{\mathbb{R}_{\eta}^{d-1} \times S^{d-2}} r^{d-k} \int_{0}^{\pi / 2} \cos { }^{d-k-2} \phi \\
\mathbf{1}_{\eta}((x, 0),(\cos \phi \cdot u,-\sin \phi)) d \phi \Xi_{k}(K, d(x, u))
\end{aligned}
$$

and the same way

$$
\begin{gathered}
\int_{0}^{r} V_{d-1}\left(\left\{x \in\left(K+\sqrt{r^{2}-y^{2}} B^{d-1}\right) \backslash K \mid\left((p(K, x), h), \frac{(x-p(K, x), y)}{\|(x-p(K, x), y)\|}\right) \in \eta\right\}\right) d y \\
=\sum_{k=0}^{d-2} \kappa_{d-k-1} \frac{d-k-1}{d-k} \int_{\mathbb{R}^{d-1} \times S^{d-2}} r^{d-k} \int_{0}^{\pi / 2} \cos ^{d-k-2} \phi \\
\mathbf{1}_{\eta}((x, h),(\cos \phi \cdot u, \sin \phi)) d \phi \Xi_{k}(K, d(x, u)) .
\end{gathered}
$$

Moreover,

$$
\begin{aligned}
\int_{0}^{h} V_{d-1}(\{x & \left.\left.\in\left(K+r B^{d-1}\right) \backslash K \mid((p(K, x), y),(u(K, x), 0)) \in \eta\right\}\right) d y \\
= & \int_{0}^{h} \sum_{k=0}^{d-2} \kappa_{d-k-1}(d-k-1) \int_{\mathbb{R}^{d-1} \times S^{d-2}} \int_{0}^{r} s^{d-k-2} \mathbf{1}_{\eta}((x, y),(u, 0)) d s \Xi_{k}(K, d(x, u)) d y \\
& =\sum_{k=0}^{d-2} \kappa_{d-k-1} \int_{0}^{h} \int_{\mathbb{R}^{d-1} \times S^{d-2}} r^{d-k-1} \mathbf{1}_{\eta}((x, y),(u, 0)) \Xi_{k}(K, d(x, u)) d y \\
& =\sum_{k=1}^{d-1} \kappa_{d-k} r^{d-k} \int_{0}^{h} \int_{\mathbb{R}^{d-1} \times S^{d-2}} \mathbf{1}_{\eta}((x, y),(u, 0)) \Xi_{k-1}(K, d(x, u)) d y .
\end{aligned}
$$


Altogether this is

$$
\begin{aligned}
\sum_{k=0}^{d-1} \kappa_{d-k} r^{d-k} \Xi_{k}(K \times[0 ; h], \eta) & r \cdot V_{d-1}\left(\left\{x \in K \mid\left((x, 0),-e_{d}\right) \in \eta\right\}\right) \\
& +\sum_{k=0}^{d-2} \kappa_{d-k-1} \frac{d-k-1}{d-k} \int_{\mathbb{R}^{d-1} \times S^{d-2}} r^{d-k} \int_{0}^{\pi / 2} \cos ^{d-k-2} \phi \\
& \mathbf{1}_{\eta}((x, 0),(\cos \phi \cdot u,-\sin \phi)) d \phi \Xi_{k}(K, d(x, u)) \\
& +\sum_{k=1}^{d-1} \kappa_{d-k} r^{d-k} \int_{0}^{h} \int_{\mathbb{R}^{d-1} \times S^{d-2}} \mathbf{1}_{\eta}((x, y),(u, 0)) \Xi_{k-1}(K, d(x, u)) d y \\
& +r \cdot V_{d-1}\left(\left\{x \in K \mid\left((x, h), e_{d}\right) \in \eta\right\}\right) \\
& +\sum_{k=0}^{d-2} \kappa_{d-k-1} \frac{d-k-1}{d-k} \int_{\mathbb{R}^{d-1} \times S^{d-2}} r^{d-k} \int_{0}^{\pi / 2} \cos ^{d-k-2} \phi \\
& \mathbf{1}_{\eta}((x, h),(\cos \phi \cdot u, \sin \phi)) d \phi \Xi_{k}(K, d(x, u)) .
\end{aligned}
$$

Since $r$ was arbitrary, the claim holds if $K$ is convex. Using the additivity of the support measures, one sees by induction that the claim holds for sets $K$ that can be obtained as the union of $n$ convex bodies, $n \in \mathbb{N}$.

Corollary 10. Let $K \subseteq \mathbb{R}^{d-1}$ be from the convex ring and $h \geq 0$. Then

$$
\begin{aligned}
V_{k}(K \times[0 ; h]) & =V_{k}(K)+h V_{k-1}(K), \quad k=1, \ldots, d-1, \\
V_{0}(K \times[0 ; h]) & =V_{0}(K), \\
\left|V_{k}\right|(K \times[0 ; h]) & \leq\left|V_{k}\right|(K)+h\left|V_{k-1}\right|(K), \quad k=1, \ldots, d-1, \\
\left|V_{0}\right|(K \times[0 ; h]) & \leq\left|V_{0}\right|(K) .
\end{aligned}
$$

Proof. The equalities (2) and (3) are immediate consequences of Lemma 9, since

$$
\frac{\kappa_{l-1}}{\kappa_{l}} \frac{l-1}{l} \int_{0}^{\pi / 2} \cos ^{l-2} \phi d \phi=\frac{1}{2}, \quad l \in \mathbb{N} .
$$

For a signed measure $\mu$ we let $\mu^{+}$denote the positive part w.r.t. the Jordan decomposition. We let $P \subseteq \mathbb{R}^{d} \times S^{d-1}$ denote the positive set of the Hahn decomposition of $\Xi_{k}(K \times[0 ; h], \cdot)$. Using the facts that $\Xi_{k}^{+}(K \times[0 ; h], \eta)=\Xi_{k}(K \times[0 ; h], \eta \cap P)$ and that $\int f(x) d \mu(x) \leq \int f(x) d \mu^{+}(x)$ for any signed measure $\mu$ defined on some measure space $(\Omega, \mathcal{A})$ and any measurable function $f: \Omega \rightarrow \mathbb{R}_{0}^{+}$and finally employing the monotonicity of measures, one concludes from Lemma 9 that

$$
\begin{aligned}
\Xi_{k}^{+}(K \times & {[0 ; h], \eta) } \\
\leq & \frac{\kappa_{d-k-1}}{\kappa_{d-k}} \frac{d-k-1}{d-k} \int_{\mathbb{R}^{d-1} \times S^{d-2}} \int_{0}^{\pi / 2} \cos ^{d-k-2} \phi \mathbf{1}_{\eta}((x, 0),(\cos \phi \cdot u,-\sin \phi)) d \phi \Xi_{k}^{+}(K, d(x, u)) \\
& +\int_{0}^{h} \int_{\mathbb{R}^{d-1} \times S^{d-2}} \mathbf{1}_{\eta}((x, y),(u, 0)) \Xi_{k-1}^{+}(K, d(x, u)) d y \\
& +\frac{\kappa_{d-k-1}}{\kappa_{d-k}} \frac{d-k-1}{d-k} \int_{\mathbb{R}^{d-1} \times S^{d-2}} \int_{0}^{\pi / 2} \cos ^{d-k-2} \phi \mathbf{1}_{\eta}((x, h),(\cos \phi \cdot u, \sin \phi)) d \phi \Xi_{k}^{+}(K, d(x, u)), \\
& k=1, \ldots, d-2,
\end{aligned}
$$


and

$$
\begin{aligned}
\Xi_{0}^{+}(K & \times[0 ; h], \eta) \\
\leq & \frac{\kappa_{d-1}}{\kappa_{d}} \frac{d-1}{d} \int_{\mathbb{R}^{d-1} \times S^{d-2}} \int_{0}^{\pi / 2} \cos ^{d-2} \phi \mathbf{1}_{\eta}((x, 0),(\cos \phi \cdot u,-\sin \phi)) d \phi \Xi_{0}^{+}(K, d(x, u)) \\
& +\frac{\kappa_{d-1}}{\kappa_{d}} \frac{d-1}{d} \int_{\mathbb{R}^{d-1} \times S^{d-2}} \int_{0}^{\pi / 2} \cos ^{d-2} \phi \mathbf{1}_{\eta}((x, h),(\cos \phi \cdot u, \sin \phi)) d \phi \Xi_{0}^{+}(K, d(x, u))
\end{aligned}
$$

for any measurable set $\eta \subseteq \mathbb{R}^{d} \times S^{d-1}$. Hence we get (4) for $k=1, \ldots, d-2$ and (5). In the case $k=d-1$ we have $V_{k}(K \times[0 ; h])=\left|V_{k}\right|(K \times[0 ; h]), V_{k}(K)=\left|V_{k}\right|(K)$ and $V_{k-1}(K)=\left|V_{k-1}\right|(K)$ and hence (4) follows from (2).

Applying Corollary 10 inductively, we get:

Corollary 11. Let $K \subseteq \mathbb{R}^{n}$ be from the convex ring, $h \geq 0$ and $N \in \mathbb{N}$. Then for $k=0, \ldots, N+n$ we have

$$
\begin{aligned}
V_{k}\left(K \times[0 ; h]^{N}\right) & =\sum_{\substack{i=\max \\
\{0, k-N\}}}^{\min \{k, n\}}\left(\begin{array}{c}
N \\
k-i
\end{array}\right) h^{k-i} V_{i}(K), \\
\left|V_{k}\right|\left(K \times[0 ; h]^{N}\right) \leq & \sum_{\substack{i=\max \\
\{0, k-N\}}}^{\min \{k, n\}}\left(\begin{array}{c}
N \\
k-i
\end{array}\right) h^{k-i}\left|V_{i}\right|(K) .
\end{aligned}
$$

\section{$3 \quad$ Pixel configuration counts}

Formula (28) of [3] says:

Lemma 12. Let $\beta \subseteq \mathbb{R}^{d}$ be a Borel set and let $U$ be a random vector distributed uniformly in $[0 ; 1]^{d}$. Then

$$
\mathbb{E} \#\left\{z \in t \mathbb{Z}^{d} \mid z+t U \in \beta\right\}=V_{d}(\beta) / t^{d} .
$$

Now we examine the pixel configuration counts of cylinder sets.

Lemma 13. Let $K \subseteq(-1 ; 1)^{d-1}$ be an arbitrary set, $-1<a<b<1$ and $0<t<\min \{1+a, 1-b\}$ and $I \in\left\{1, \ldots, 2^{\left(2^{d}\right)}\right\}$ the number of a pixel configuration $\left(B_{I}, W_{I}\right)$. If there is at least one integer multiple of $t$ in $[a ; b]$, then $N_{t, I}(K \times[a ; b])$ equals

- $N_{t, i}(K)$ if there is a d-1-dimensional pixel configuration $\left(B_{i}, W_{i}\right)$ fulfilling $\left(B_{I}=B_{i} \times\{0\} \neq \emptyset\right.$ and $\left.W_{I}=W_{i} \times\{0\} \cup\{0,1\}^{d-1} \times\{1\}\right)$ or $\left(B_{I}=B_{i} \times\{1\} \neq \emptyset\right.$ and $\left.W_{I}=W_{i} \times\{1\} \cup\{0,1\}^{d-1} \times\{0\}\right)$,

- $(p-1) \cdot N_{t, i}(K)$ if $B_{I}=B_{i} \times\{0,1\} \neq \emptyset$ and $W_{I}=W_{i} \times\{0,1\}$, where $p:=\#\{z \in \mathbb{Z} \mid t z \in[a ; b]\}$,

- $(q-p-2) \cdot(q-1)^{d-1}+(p+1) \cdot N_{t, i}(K)$ if $B_{I}=\emptyset, W_{I}=\{0,1\}^{d}, B_{i}=\emptyset$ and $W_{i}=\{0,1\}^{d-1}$, where $q:=\#\{z \in \mathbb{Z} \mid t z \in[-1 ; 1]\}$ is the number of pixels in $[-1 ; 1]$,

- 0 otherwise.

If there is no integer multiple of $t$ in $[a ; b]$, then we have $N_{t, I}(K \times[a ; b])=(q-1)^{d}$ if $B_{I}=\emptyset$ and $W_{I}=\{0,1\}^{d}$ and we have $N_{t, I}(K \times[a ; b])=0$ otherwise.

Proof. Assume that there is an integer multiple of $t$ in $[a ; b]$ and that $B_{I}=B_{i} \times\{0,1\} \neq \emptyset$ and $W_{I}=W_{i} \times\{0,1\}$. Then for all $x \in t \mathbb{Z}^{d-1}$ and $y \in t \mathbb{Z}$ the conditions

$$
(x, y)+t B_{I} \subseteq K \times[a ; b] \wedge(x, y)+t W_{I} \subseteq(K \times[a ; b])^{C}
$$


and

$$
x+t B_{i} \subseteq K \wedge x+t W_{i} \subseteq K^{C} \wedge\{y, y+t\} \subseteq[a ; b]
$$

are equivalent. Hence

$$
\begin{aligned}
N_{t, I} & (K \times[a ; b]) \\
\quad & =\#\left(\left\{x \in t \mathbb{Z}^{d-1} \cap[-1 ; 1-t]^{d-1} \mid x+t B_{i} \subseteq K \wedge x+t W_{i} \subseteq K^{C}\right\} \times\{y \in t \mathbb{Z} \mid\{y, y+t\} \subseteq[a ; b]\}\right) \\
\quad & =N_{t, i}(K) \cdot(p-1) .
\end{aligned}
$$

The other assertions of the lemma follow similarly.

We now fix the enumeration of the equivalence classes of $2 \times 2$-pixel configurations. Since $\nu_{2}=6$, the numbers $0, \ldots, 5$ will be used to enumerate these equivalence classes. For $j \in\{0,1,3,4\}$ there is exactly one equivalence class whose configurations have $j$ black (foreground) pixels and $4-j$ white pixels. We denote this class by $j$. There are exactly two equivalence classes whose pixel configurations have two foreground and two background pixels. We call the class with two neighboring foreground pixels number 2 and the class with two opposite foreground pixels number 5.

Next we assign names to some equivalence classes of $2^{d}$-pixel configurations, $d \geq 2$. If $\xi \in\{0, \ldots, d-2\}$ is a number and $(B, W)$ is a $2 \times 2$-pixel-configuration of equivalence class $\iota \in\{0, \ldots, 5\}$, we denote the equivalence class to which the $2^{d}$-pixel-configuration

$$
\left(B \times\{0,1\}^{\xi} \times\{0\}^{d-\xi-2}, W \times\{0,1\}^{\xi} \times\{0\}^{d-\xi-2} \cup\{0,1\}^{\xi+2} \times\left(\{0,1\}^{d-\xi-2} \backslash\{(0, \ldots, 0)\}\right)\right)
$$

belongs by $\langle\iota: \xi: d\rangle$. We notice that an equivalence class may have one name, no name or several names. In images of cylinder sets with a 2-dimensional base only pixel configurations belonging to equivalence classes which have a name can occur. More precisely, we have the following corollary, which follows from Lemma 13 by induction.

Corollary 14. If $\tilde{N}_{t, j}\left(K \times\left[a_{1} ; b_{1}\right] \times\left[a_{2} ; b_{2}\right] \times \cdots \times\left[a_{d-2} ; b_{d-2}\right]\right) \neq 0$ for a set $K \subseteq[-1 ; 1]^{2}$, numbers $a_{1}<b_{1}, \ldots, a_{d-2}<b_{d-2}$, some $t>0$ and some equivalence class $j$, then $j$ is one of the following:

$$
\begin{aligned}
& \langle 1: \xi: d\rangle,\langle 3: \xi: d\rangle,\langle 5: \xi: d\rangle, \quad \xi \in\{0, \ldots, d-2\}, \\
& \langle 0: 0: d\rangle,\langle 2: d-2: d\rangle,\langle 4: d-2: d\rangle .
\end{aligned}
$$

Theorem 15. For $t \in(0 ; 1 / 3), \xi \in\{-2, \ldots, d-2\}$ there are functions $c_{t, \xi}:(t ; 1-2 t)^{d-2} \rightarrow \mathbb{R}_{0}^{+}$such that

$$
\begin{aligned}
\mathbb{E} \tilde{N}_{t,\langle 1: \xi: d\rangle}\left(X \times\left[0 ; h_{1}\right] \times \cdots \times\left[0 ; h_{d-2}\right]+t U\right)= & c_{t, \xi}\left(h_{1}, \ldots, h_{d-2}\right) \cdot \mathbb{E} \tilde{N}_{t, 1}(X) \\
& +c_{t, \xi-1}\left(h_{1}, \ldots, h_{d-2}\right) \cdot \mathbb{E} \tilde{N}_{t, 2}(X) \\
& +c_{t, \xi-2}\left(h_{1}, \ldots, h_{d-2}\right) \cdot \mathbb{E} \tilde{N}_{t, 4}(X), \xi \in\{0, \ldots, d-2\}, \\
\mathbb{E} \tilde{N}_{t,\langle 2: d-2: d\rangle}\left(X \times\left[0 ; h_{1}\right] \times \cdots \times\left[0 ; h_{d-2}\right]+t U\right)= & c_{t, d-2}\left(h_{1}, \ldots, h_{d-2}\right) \cdot \mathbb{E} \tilde{N}_{t, 2}(X) \\
& +c_{t, d-3}\left(h_{1}, \ldots, h_{d-2}\right) \cdot \mathbb{E} \tilde{N}_{t, 4}(X) \\
\mathbb{E} \tilde{N}_{t,\langle 4: d-2: d\rangle}\left(X \times\left[0 ; h_{1}\right] \times \cdots \times\left[0 ; h_{d-2}\right]+t U\right)= & c_{t, d-2}\left(h_{1}, \ldots, h_{d-2}\right) \cdot \mathbb{E} \tilde{N}_{t, 4}(X) \\
\mathbb{E} \tilde{N}_{t,\langle 3: \xi: d\rangle}\left(X \times\left[0 ; h_{1}\right] \times \cdots \times\left[0 ; h_{d-2}\right]+t U\right)= & c_{t, \xi}\left(h_{1}, \ldots, h_{d-2}\right) \cdot \mathbb{E} \tilde{N}_{t, 3}(X), \xi \in\{0, \ldots, d-2\}, \\
\mathbb{E} \tilde{N}_{t,\langle 5: \xi: d\rangle}\left(X \times\left[0 ; h_{1}\right] \times \cdots \times\left[0 ; h_{d-2}\right]+t U\right)= & c_{t, \xi}\left(h_{1}, \ldots, h_{d-2}\right) \cdot \mathbb{E} \tilde{N}_{t, 5}(X), \xi \in\{0, \ldots, d-2\},
\end{aligned}
$$

for any random compact set $X \subseteq(-1 ; 1)^{2}$ and $h_{1}, \ldots, h_{d-2} \in(t ; 1-2 t)$, where $U$ is a random vector distributed uniformly on $\{(0,0)\} \times[0 ; 1]^{d-2}$. These functions are monotonically increasing in all arguments and fulfill $c_{t, \xi} \equiv 0$ if $\xi<0$ and

$$
c_{t, \xi}(h, \ldots, h)=\left(\begin{array}{c}
d-2 \\
\xi
\end{array}\right) 2^{d-2-\xi}\left(\frac{h}{t}-1\right)^{\xi}
$$

for $h \in(t, 1-2 t)$ if $\xi \geq 0$. 
Proof. We define the functions $c_{t, \xi}$ recursively. If $d=2$, we put $c_{t, \xi}=1$ for $\xi=0$ and $c_{t, \xi}=0$ for $\xi<0$. If $d>2$ we put $c_{t,-2}\left(h_{1}, \ldots, h_{d-2}\right)=0$,

$$
c_{t, \xi}\left(h_{1}, \ldots, h_{d-2}\right)=\left(\frac{h_{d-2}}{t}-1\right) c_{t, \xi-1}\left(h_{1}, \ldots, h_{d-3}\right)+2 c_{t, \xi}\left(h_{1}, \ldots, h_{d-3}\right), \quad \xi \in\{-1, \ldots, d-3\},
$$

and

$$
c_{t, d-2}\left(h_{1}, \ldots, h_{d-2}\right)=\left(\frac{h_{d-2}}{t}-1\right) c_{t, d-3}\left(h_{1}, \ldots, h_{d-3}\right) .
$$

Assume $d \geq 3$ now. For fixed $\iota \in\{1, \ldots, 5\}$ and $\xi \in\{0, \ldots, d-2\}$ all pixel configurations, that belong to class $\langle\iota: \xi: d\rangle$ and may occur in images of cylinder sets $K \times[a ; b], K \subseteq(-1 ; 1)^{d-1},-1<a<b<1$, are of one of the following types:

- $\left(\tilde{B} \times\{0\}, \tilde{W} \times\{0\} \cup\{0,1\}^{d-1} \times\{1\}\right)$ and $\left(\tilde{B} \times\{1\}, \tilde{W} \times\{1\} \cup\{0,1\}^{d-1} \times\{0\}\right)$, where $(\tilde{B}, \tilde{W})$ is a pixel configuration of class $\langle\iota: \xi: d-1\rangle$ iff $\xi<d-2$,

- $\left(\tilde{B} \times\{0\}, \tilde{W} \times\{0\} \cup\{0,1\}^{d-1} \times\{1\}\right)$ and $\left(\tilde{B} \times\{1\}, \tilde{W} \times\{1\} \cup\{0,1\}^{d-1} \times\{0\}\right)$, where $(\tilde{B}, \tilde{W})$ is a pixel configuration of class $\langle 2 \iota: \xi-1: d-1\rangle$ iff $\iota \in\{1,2\}$ and $\xi>0$ (notice that these configurations were already mentioned in the first point iff $\xi<d-2$ ),

- $(\tilde{B} \times\{0,1\}, \tilde{W} \times\{0,1\})$, where $(\tilde{B}, \tilde{W})$ is a pixel configuration of class $\langle\iota: \xi-1: d-1\rangle$ iff $\xi>0$, and

- $(\tilde{B} \times\{0,1\}, \tilde{W} \times\{0,1\})$, where $(\tilde{B}, \tilde{W})$ is a pixel configuration of class $\langle\iota / 2: \xi: d-1\rangle$ iff $\iota \in\{2,4\}$ and $\xi<d-2$ (notice that these configurations were already mentioned in the third point iff $\xi>0$ ).

It is easy to see that all these configurations belong to class $\langle\iota: \xi: d\rangle$. In order to prove the reverse statement, let $(B, W)$ be a pixel configuration of class $\langle\iota: \xi: d\rangle$. Then there is an isometry $g: \mathbb{R}^{d} \rightarrow \mathbb{R}^{d}$ with

$$
g(B)=B_{\iota} \times\{0,1\}^{\xi} \times\{0\}^{d-\xi-2}
$$

and

$$
g(W)=W_{\iota} \times\{0,1\}^{\xi} \times\{0\}^{d-\xi-2} \cup\{0,1\}^{\xi+2} \times\left(\{0,1\}^{d-\xi-2} \backslash\{0\}^{d-\xi-2}\right),
$$

where $\left(B_{\iota}, W_{\iota}\right)$ is a $2 \times 2$-pixel configuration of class $\iota$. Since $g$ leaves $\{0,1\}^{d}$ invariant, there must be some $\ell \in\{1, \ldots, d\}$ with $g\left(e_{d}\right)-g(0)= \pm e_{\ell}$. Distinguishing cases w.r.t. $\ell$, one sees that $(B, W)$ really is of one of the four types mentioned above. We demonstrate this for the case $\ell=1$. By Lemma 13 there are sets $\tilde{B} \subseteq\{0,1\}^{d-1}, B^{\prime} \subseteq\{0,1\}$ with $B=\tilde{B} \times B^{\prime}$ and hence there are sets $A^{(1)} \subseteq\{0,1\}$ and $A^{(2)} \subseteq\{0,1\}^{d-1}$ with

$$
A^{(1)} \times A^{(2)}=g(B)=B_{\iota} \times\{0,1\}^{\xi} \times\{0\}^{d-\xi-2} .
$$

Thus $\iota \neq 3$ and $\iota \neq 5$. We have to consider several subcases and show here only the subcase $\iota=1, \xi>0$. Then $B^{\prime}=\{0\}$ or $B^{\prime}=\{1\}$. Moreover, let $h: \mathbb{R}^{d} \rightarrow \mathbb{R}^{d}$ be the map that moves the first component to the $d$-th, the $d$-th component to the $\xi+2$-nd, the $\xi+2$-nd to the first and leaves all other components invariant. We have $(h \circ g)\left(e_{d}\right)-(h \circ g)(0)= \pm e_{d}$, w.l.o.g. $(h \circ g)\left(e_{d}\right)-(h \circ g)(0)=+e_{d}$. Then $(h \circ g)\left(\mathbb{R}^{d-1}\right) \subseteq \mathbb{R}^{d-1}$ and so we can consider the map

$$
f: \mathbb{R}^{d-1} \rightarrow \mathbb{R}^{d-1}, x \mapsto(h \circ g)(x) .
$$

Now $f(\tilde{B})=\{0,1\} \times\{0\} \times\{0,1\}^{\xi-1} \times\{0\}^{d-\xi-2}$, the $2 \times 2$-pixel-configuration $(\{0,1\} \times\{0\},\{0,1\} \times\{1\})$ is of equivalence class 2 and $f$ is an isometry. So $(\tilde{B}, \tilde{W})$ is of class $\langle 2: \xi-1: d-1\rangle$. Treating all remaining cases in a similar way, one sees that $(B, W)$ is of one of the four types mentioned above.

Lemma 12 yields $\mathbb{E} \#\left\{z \in \mathbb{Z} \mid t z \in\left[t U_{2} ; h_{d-2}+t U_{2}\right]\right\}=\frac{h_{d-2}}{t}$, where $U_{2}$ is a random number distributed uniformly on $[0 ; 1]$. We let $U_{1}$ denote a random vector distributed uniformly on $\{(0,0)\} \times[0 ; 1]^{d-3} \subseteq \mathbb{R}^{d-1}$. Now Lemma 13 gives

$$
\begin{aligned}
\mathbb{E} \tilde{N}_{t,\langle\iota: d-2: d\rangle}(X & \left.\times\left[0 ; h_{1}\right] \times \cdots \times\left[0 ; h_{d-2}\right]+t U\right) \\
= & 2 \cdot \mathbb{E} \tilde{N}_{t,\langle 2 \iota: d-3: d-1\rangle}\left(X \times\left[0 ; h_{1}\right] \times \cdots \times\left[0 ; h_{d-3}\right]+t U_{1}\right) \\
& +\left(\frac{h_{d-2}}{t}-1\right) \cdot \mathbb{E} \tilde{N}_{t,\langle\iota: d-3: d-1\rangle}\left(X \times\left[0 ; h_{1}\right] \times \cdots \times\left[0 ; h_{d-3}\right]+t U_{1}\right), \quad \iota \in\{1,2\},
\end{aligned}
$$




$$
\begin{aligned}
& \mathbb{E} \tilde{N}_{t,\langle\iota: d-2: d\rangle}(\left.X \times\left[0 ; h_{1}\right] \times \cdots \times\left[0 ; h_{d-2}\right]+t U\right) \\
&=\left(\frac{h_{d-2}}{t}-1\right) \cdot \mathbb{E} \tilde{N}_{t,\langle\iota: d-3: d-1\rangle}\left(X \times\left[0 ; h_{1}\right] \times \cdots \times\left[0 ; h_{d-3}\right]+t U_{1}\right), \quad \iota \in\{3,4,5\}, \\
& \mathbb{E} \tilde{N}_{t,\langle\iota: 0: d\rangle}\left(X \times\left[0 ; h_{1}\right] \times \cdots \times\left[0 ; h_{d-2}\right]+t U\right) \\
&=2 \cdot \mathbb{E} \tilde{N}_{t,\langle\iota: 0: d-1\rangle}\left(X \times\left[0 ; h_{1}\right] \times \cdots \times\left[0 ; h_{d-3}\right]+t U_{1}\right), \quad \iota \in\{1,3,5\}, \\
& \mathbb{E} \tilde{N}_{t,\langle\iota: \xi: d\rangle}\left(X \times\left[0 ; h_{1}\right] \times \cdots \times\left[0 ; h_{d-2}\right]+t U\right) \\
&=2 \cdot \mathbb{E} \tilde{N}_{t,\langle\iota: \xi: d-1\rangle}\left(X \times\left[0 ; h_{1}\right] \times \cdots \times\left[0 ; h_{d-3}\right]+t U_{1}\right) \\
& \quad+\left(\frac{h_{d-2}}{t}-1\right) \cdot \mathbb{E} \tilde{N}_{t,\langle\iota: \xi-1: d-1\rangle}\left(X \times\left[0 ; h_{1}\right] \times \cdots \times\left[0 ; h_{d-3}\right]+t U_{1}\right), \\
& \iota \in\{1,3,5\}, \xi \in\{1, \ldots, d-3\} .
\end{aligned}
$$

Now the assertions follow by an elementary induction.

\section{Proof of the Main Results}

Recall the enumeration of equivalence classes of $2 \times 2$-pixel configurations from page 9 .

Lemma 16. If the worst case asymptotic bias of an estimator of type

$$
\hat{\gamma}=\sum_{j=0}^{5} \lambda_{j}(t) \tilde{N}_{t, j}
$$

is finite on $\left\{K \in \mathcal{P}^{(2)}\left|K \subseteq(-1 ; 1)^{2},\right| V_{0} \mid(K) \leq 2\right\}$, then

$$
\begin{aligned}
\left|\lambda_{0}(t)\right|,\left|\lambda_{4}(t)\right| & \in O\left(t^{2}\right), \\
\left|\lambda_{2}(t)\right| & \in O(t), \\
\left|\lambda_{1}(t)\right|,\left|\lambda_{3}(t)\right|,\left|\lambda_{5}(t)\right| & \in o\left(t^{0}\right) .
\end{aligned}
$$

It seems to be impossible to write Lemma 16 and its proof in such a way that the essential ideas become clearly visible and are not covered by technical details. Hence we comment on the essential ideas before we come to the proof.

The equation (10) is the central point in Lemma 16, while (8) and (9) are technical details: First (8) and (9) are the conditions that experts would have expected anyway, while they probably would have conjectured that we get only $O\left(t^{0}\right)$ in (10). Moreover, most of the estimators for the Euler characteristic we found in the literature fulfill (8) and (9) and violate (10).

In the proof below we will show

$$
\mathbb{E} \tilde{N}_{t, 1}(A+t U)=\mathbb{E} \tilde{N}_{t, 3}(A+t U)+4+2 \mathbb{E} \tilde{N}_{t, 5}(A+t U)
$$

for certain parallelograms $A$ and random vectors $U$. Using different methods one could show

$$
\tilde{N}_{t, 1}\left(A^{\prime}\right)=\tilde{N}_{t, 3}\left(A^{\prime}\right)+4+2 \tilde{N}_{t, 5}\left(A^{\prime}\right)
$$

for compact, simply connected sets $A^{\prime}$ that fulfill some weak regularity condition. Pixel configurations of class $1,2,3$ and 5 can only occur on the boundary of the observed set $K \subseteq \mathbb{R}^{d}$. Given $K$ fulfills certain regularity conditions (which we do not know exactly) and the lattice distance $t$ is sufficiently small, each pixel configuration of class $1,2,3$ and 5 belongs to a uniquely determined connected component of bd $K$. Obviously, (11) also holds for the number of occurrences of pixel configurations on the outer boundary of each connected component of the more complex set $K$. If we consider a hole of shape $A^{\prime}$ within $K$, (11) gives

$$
\tilde{N}_{t, 3}=\tilde{N}_{t, 1}+4+2 \tilde{N}_{t, 5}
$$


for the number of pixel configurations on the (outer) boundary of that hole, since black and white pixels are interchanged, hence pixel configurations of class 1 become pixel configurations of class 3 and the other way round, while pixel configurations of class 5 stay in that class. So $\tilde{N}_{t, 1}(K)-\tilde{N}_{t, 3}(K)$ equals four times the Euler characteristic of a set $K \subseteq \mathbb{R}^{2}$, provided that $\tilde{N}_{t, 5}(K)=0$ and that certain regularity conditions are fulfilled. If, however, the number of occurrences of pixel configurations of type 5 is large, the difference $\tilde{N}_{t, 1}-\tilde{N}_{t, 3}$ may be far from 0 , even if $\left|V_{0}\right|(K)$ is small. One could correct for this effect by adding to $\tilde{N}_{t, 1}-\tilde{N}_{t, 3}$ twice the number of pixel configurations of type 5 lying on "inner" boundary parts and subtracting twice the number of pixel configurations of type 5 lying on "outer" boundary parts. However, these numbers are not observed (only their sum is observed) and hence the only way to prevent the estimator from returning values whose absolute values are huge compared to $\left|V_{0}\right|(K)$ is to choose the weights $\lambda_{1}(t), \lambda_{3}(t)$ and $\lambda_{5}(t)$ unnaturally close to 0 .

Proof of Lemma 16. We find it convenient to start with the proof of central point (10), even though this means that we cannot present the proof in logical order. So assume we have already shown (8), (9),

$$
\left|\lambda_{1}(t)\right| \in O\left(t^{0}\right) \text { and }\left|\lambda_{1}(t)+\lambda_{3}(t)\right| \in O(t) .
$$

For $m_{1} \in(0 ; 1)$ and $m_{2} \in(1 ; \infty)$ consider the set

$$
A:=\left\{(x, y) \in \mathbb{R}^{2} \mid m_{1}\left(x+\frac{1}{3}\right) \leq y+\frac{1}{3} \leq m_{2}\left(x+\frac{1}{3}\right), m_{2}\left(x-\frac{1}{3}\right) \leq y-\frac{1}{3} \leq m_{1}\left(x-\frac{1}{3}\right)\right\} .
$$

As obviously

$$
\begin{array}{r}
\sup _{m_{1}, m_{2}} \limsup _{t \rightarrow 0} \frac{\mathbb{E} \tilde{N}_{t, 0}(A+t U)}{t^{-2}}<\infty, \\
\sup _{m_{1}, m_{2}} \limsup _{t \rightarrow 0} \frac{\mathbb{E} \tilde{N}_{t, 4}(A+t U)}{t^{-2}}<\infty \\
\text { and } \sup _{m_{1}, m_{2}} \limsup _{t \rightarrow 0} \frac{\mathbb{E} \tilde{N}_{t, 2}(A+t U)}{t^{-1}}<\infty,
\end{array}
$$

where $U$ is a random vector distributed uniformly on $[0,1]^{d}$, we get

$$
\begin{aligned}
\sup _{m_{1}, m_{2}} \limsup _{t \rightarrow 0}\left|\mathbb{E} \hat{\gamma}(A+t U)-\sum_{j=1,3,5} \lambda_{j}(t) \mathbb{E} \tilde{N}_{t, j}(A+t U)\right| \\
=\sup _{m_{1}, m_{2}} \limsup _{t \rightarrow 0}\left|\sum_{j=0,2,4} \lambda_{j}(t) \mathbb{E} \tilde{N}_{t, j}(A+t U)\right| \\
<\infty .
\end{aligned}
$$

In order to compute $\mathbb{E} \tilde{N}_{t, 5}(A+t U)$, we put $B:=\{(0,0),(1,1)\}$ and $W:=\{(0,1),(1,0)\}$ and observe (see Figure 1)

$$
\begin{aligned}
C_{t}:= & \left\{v \in \mathbb{R}^{2} \mid v+t B \subseteq A, v+t W \subseteq A^{C}\right\} \\
= & \left\{(x, y) \in \mathbb{R}^{2} \mid m_{1}\left(x+\frac{1}{3}\right) \leq y+\frac{1}{3} \leq m_{2}\left(x+\frac{1}{3}\right), m_{2}\left(x+\frac{1}{3}\right)<y+\frac{1}{3}+t, y+\frac{1}{3}<m_{1}\left(x+\frac{1}{3}+t\right)\right\} \\
\cup\left\{(x, y) \in \mathbb{R}^{2} \mid\right. & m_{2}\left(x-\frac{1}{3}+t\right) \leq y-\frac{1}{3}+t \leq m_{1}\left(x-\frac{1}{3}+t\right), m_{1}\left(x-\frac{1}{3}\right)<y-\frac{1}{3}+t, \\
& \left.\quad y-\frac{1}{3}<m_{2}\left(x-\frac{1}{3}+t\right)\right\}
\end{aligned}
$$

for $t \in\left(0 ; \frac{1}{3} \min \left\{2-2 m_{1}, \frac{2 m_{2}-2}{m_{2}}\right\}\right)$. Since this union is disjoint for $t \in\left(0 ; \frac{1}{3} \min \left\{\frac{2-2 m_{1}}{m_{1}+1}, \frac{2 m_{2}-2}{m_{2}+1}\right\}\right)$, its area is $2 \cdot t^{2} \cdot s$, where $s:=m_{1} /\left(m_{2}-m_{1}\right)$. Hence Lemma 12 gives $\mathbb{E} \tilde{N}_{t, 5}(A+t U)=2 s$ for sufficiently small $t>0$. Similar arguments yield

$$
\mathbb{E} \tilde{N}_{t, 1}(A+t U)=2+2 \frac{m_{1}\left(\frac{2 m_{2}-2}{3 t}-m_{2}\right)+\left(\frac{2-2 m_{1}}{3 t}-1\right)+m_{2}}{m_{2}-m_{1}}
$$




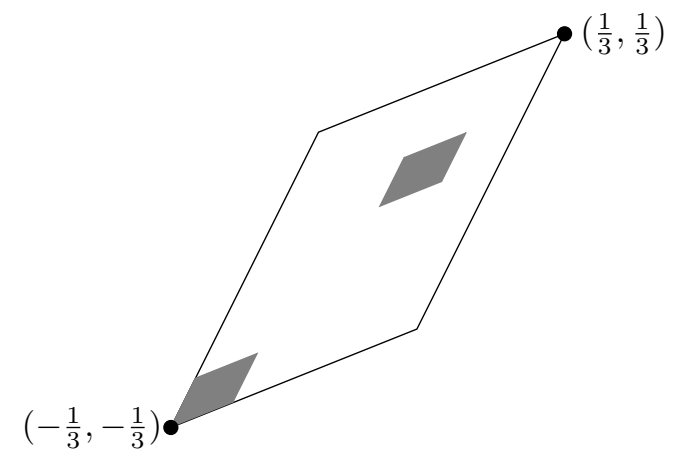

Figure 1: The set $A$ (white) and the set $C_{t}$ (grey)

and

$$
\mathbb{E} \tilde{N}_{t, 3}(A+t U)=2 \frac{m_{1}\left(\frac{2 m_{2}-2}{3 t}-m_{2}\right)+\left(\frac{2-2 m_{1}}{3 t}-1\right)-m_{1}}{m_{2}-m_{1}} .
$$

Hence

$$
\mathbb{E} \tilde{N}_{t, 1}(A+t U)=\mathbb{E} \tilde{N}_{t, 3}(A+t U)+4+2 \mathbb{E} \tilde{N}_{t, 5}(A+t U)
$$

and using (18) one obtains

$$
\sup _{m_{1}, m_{2}} \limsup _{t \rightarrow 0}\left|\mathbb{E} \hat{\gamma}(A+t U)-\left(\lambda_{1}(t) \cdot(4+4 s)+\left(\lambda_{1}(t)+\lambda_{3}(t)\right) \mathbb{E} \tilde{N}_{t, 3}(A+t U)+\lambda_{5}(t) \cdot 2 s\right)\right|<\infty
$$

which gives

$$
\sup _{m_{1}, m_{2}} \limsup _{t \rightarrow 0}\left|\mathbb{E} \hat{\gamma}(A+t U)-\left(\lambda_{1}(t) \cdot(4 s)+\lambda_{5}(t) \cdot(2 s)\right)\right|<\infty
$$

since

$$
\sup _{m_{1}, m_{2}} \limsup _{t \rightarrow 0} \frac{\mathbb{E} \tilde{N}_{t, 3}(A+t U)}{t^{-1}}<\infty .
$$

Because $s$ may be arbitrarily large, the assumption that the worst case asymptotic bias of $\hat{\gamma}$ is finite yields $\left|2 \lambda_{1}(t)+\lambda_{5}(t)\right| \in o\left(t^{0}\right)$.

Put $A_{1}:=[-1 / 2 ; 1 / 2]^{2} \backslash \operatorname{int} A$ (see Figure 2). Observing that the cells of $t \mathbb{Z}^{2}$ having (exactly) one vertex in $A_{1}+t U$ are precisely the cells having three vertices in $A+t U$ and four cells containing the corners of $[-1 / 2 ; 1 / 2]^{2}+t U$, that the cells having three vertices in $A_{1}+t U$ are precisely the cells having one vertex in $A+t U$ and that the cells having two opposite vertices in $A_{1}+t U$ are precisely the cells having two opposite vertices in $A+t U$, we conclude from (20)

$$
\mathbb{E} \tilde{N}_{t, 3}\left(A_{1}+t U\right)=\mathbb{E} \tilde{N}_{t, 1}\left(A_{1}+t U\right)+2 \mathbb{E} \tilde{N}_{t, 5}\left(A_{1}+t U\right) .
$$

Similar as above we conclude

$$
\sup _{m_{1}, m_{2}} \limsup _{t \rightarrow 0}\left|\mathbb{E} \hat{\gamma}\left(A_{1}+t U\right)-\left(\lambda_{3}(t) \cdot(4 s)+\lambda_{5}(t) \cdot(2 s)\right)\right|<\infty
$$

and hence we obtain arbitrary large asymptotic biases unless $\left|2 \lambda_{3}(t)+\lambda_{5}(t)\right| \in o\left(t^{0}\right)$.

From (13), $\left|2 \lambda_{1}(t)+\lambda_{5}(t)\right| \in o\left(t^{0}\right)$ and $\left|2 \lambda_{3}(t)+\lambda_{5}(t)\right| \in o\left(t^{0}\right)$, one concludes by Lemma 17 below that $\left|\lambda_{1}(t)\right| \in o\left(t^{0}\right),\left|\lambda_{3}(t)\right| \in o\left(t^{0}\right)$ and $\left|\lambda_{5}(t)\right| \in o\left(t^{0}\right)$.

It remains to show (8), (9) and (13). 

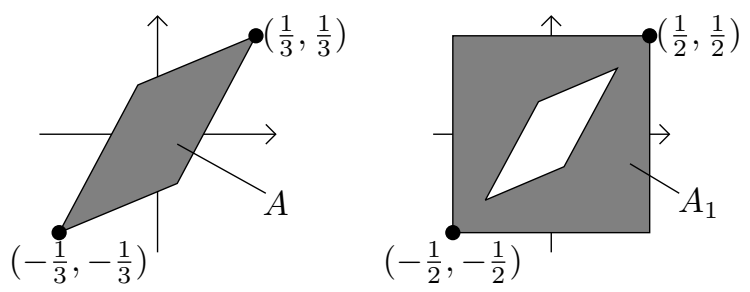

Figure 2: The sets $A$ and $A_{1}$

We let $r(t):=\#\left(t \mathbb{Z}^{2} \cap[-1,1-t]^{2}\right)$ denote the number of $2 \times 2$-pixel-configurations in our observation window at lattice distance $t$. Obviously $\lim _{t \rightarrow 0} r(t) / t^{-2}=4$.

Consider an axes-parallel square $Q_{a} \subseteq(-1 ; 1)^{2}$ of side-length $a \in(0 ; 1 / 2)$. If then $t<a$ is smaller than half the (minimal) distance between (a point from) $Q_{a}$ and (a point from) bd([-1;1 $\left.]^{2}\right)$, one has by Lemma 12

$$
\begin{gathered}
\mathbb{E} \hat{\gamma}\left(Q_{a}+t U\right)=\left(r(t)-\left(\frac{a}{t}+1\right)^{2}\right) \lambda_{0}(t)+4 \lambda_{1}(t)+\left(4 \frac{a}{t}-4\right) \lambda_{2}(t)+\left(\frac{a}{t}-1\right)^{2} \lambda_{4}(t) \\
=a^{2}\left[\frac{-1}{t^{2}} \lambda_{0}(t)+\frac{1}{t^{2}} \lambda_{4}(t)\right]+a\left[\frac{-2}{t} \lambda_{0}(t)+\frac{4}{t} \lambda_{2}(t)+\frac{-2}{t} \lambda_{4}(t)\right] \\
+(r(t)-1) \lambda_{0}(t)+4 \lambda_{1}(t)-4 \lambda_{2}(t)+\lambda_{4}(t) .
\end{gathered}
$$

Let $\tilde{Q}_{a} \subseteq(-1 ; 1)^{2}$ denote a set consisting of two disjoint copies of $Q_{a}$. If then $t<a$ is smaller than half the distance between $\tilde{Q}_{a}$ and $\mathrm{bd}\left([-1 ; 1]^{2}\right)$ and smaller than the distance between the two connected components of $\tilde{Q}_{a}$ devided by $\sqrt{2}$, we get

$$
\begin{aligned}
\mathbb{E} \hat{\gamma}\left(\tilde{Q}_{a}+t U\right)= & a^{2}\left[\frac{-2}{t^{2}} \lambda_{0}(t)+\frac{2}{t^{2}} \lambda_{4}(t)\right]+a\left[\frac{-4}{t} \lambda_{0}(t)+\frac{8}{t} \lambda_{2}(t)+\frac{-4}{t} \lambda_{4}(t)\right] \\
& +(r(t)-2) \lambda_{0}(t)+8 \lambda_{1}(t)-8 \lambda_{2}(t)+2 \lambda_{4}(t)
\end{aligned}
$$

Since

$$
2 \mathbb{E} \hat{\gamma}\left(Q_{a}+t U\right)-\mathbb{E} \hat{\gamma}\left(\tilde{Q}_{a}+t U\right)=r(t) \lambda_{0}(t)
$$

does not diverge, we get $\left|\lambda_{0}(t)\right| \in O\left(t^{2}\right)$. Because $\mathbb{E} \hat{\gamma}\left(Q_{a}+t U\right)$ does not diverge for any $a$, we conclude $\left|\lambda_{4}(t)\right| \in O\left(t^{2}\right),\left|\lambda_{2}(t)\right| \in O(t)$ and $\left|\lambda_{1}(t)\right| \in O\left(t^{0}\right)$.

Now consider a rectangular triangle $D_{a} \subseteq(-1 ; 1)^{2}$ with axes-parallel cathetus both of length $a \in(0 ; 1)$. If $t<a$ is smaller than half the distance between $D_{a}$ and $\mathrm{bd}\left([-1 ; 1]^{2}\right)$, one has

$$
\begin{aligned}
\mathbb{E} \hat{\gamma}\left(D_{a}+t U\right)= & \left(\frac{5}{2}+\frac{a}{t}\right) \lambda_{1}(t)+\left(2 \frac{a}{t}-3\right) \lambda_{2}(t)+\left(\frac{a}{t}-\frac{3}{2}\right) \lambda_{3}(t) \\
& +\left(\frac{a^{2}}{2 t^{2}}-\frac{2 a}{t}+2\right) \lambda_{4}(t)+\left(r(t)-\frac{a^{2}}{2 t^{2}}-2 \frac{a}{t}\right) \lambda_{0}(t)
\end{aligned}
$$

We conclude $\left|\left(\frac{5}{2}+\frac{a}{t}\right) \lambda_{1}(t)+\left(\frac{a}{t}-\frac{3}{2}\right) \lambda_{3}(t)\right| \in O\left(t^{0}\right)$ and thus $\left|\lambda_{3}(t)+\lambda_{1}(t)\right| \in O(t)$.

Lemma 17. Let $m \in \mathbb{Z}$ and $f_{1}, f_{2}, f_{3}: \mathbb{R}^{+} \rightarrow \mathbb{R}$ be three functions with $\left|f_{1}(t)+f_{2}(t)\right| \in o\left(t^{m}\right), \mid f_{1}(t)+$ $f_{3}(t) \mid \in o\left(t^{m}\right)$ and $\left|f_{2}(t)+f_{3}(t)\right| \in o\left(t^{m}\right)$ as $t \rightarrow 0$. Then $\left|f_{1}(t)\right|,\left|f_{2}(t)\right|,\left|f_{3}(t)\right| \in o\left(t^{m}\right)$.

Lemma 18. If the worst case asymptotic bias of an estimator of type

$$
\hat{\gamma}=\sum_{j=0}^{\nu_{d}-1} \lambda_{j}(t) \tilde{N}_{t, j}
$$

is finite on

$$
\left\{K \in \mathcal{P}^{(d)}\left|K \subseteq(-1 ; 1)^{d},\right| V_{k} \mid(K) \leq 2\left(\begin{array}{l}
d \\
k
\end{array}\right) \forall_{k \in\{0, \ldots, d\}}\right\}
$$

then $\lambda_{\langle 0: 0: d\rangle}(t) \in O\left(t^{d}\right), \lambda_{\langle 2: d-2: d\rangle}(t) \in O\left(t^{d-1}\right), \lambda_{\langle 4: d-2: d\rangle}(t) \in O\left(t^{d}\right)$ and

$$
\left|\lambda_{\langle\iota: \xi: d\rangle}(t)\right| \in o\left(t^{\xi}\right), \iota \in\{1,3,5\}, \xi \in\{0, \ldots, d-2\} .
$$


Proof. Considering axes-parallel cubes and sets that are the union of two disjoint axes-parallel cubes one gets

$$
\left|\lambda_{\langle 0: \xi: d\rangle}(t)\right| \in O\left(t^{d}\right),\left|\lambda_{\langle 1: \xi: d\rangle}(t)\right| \in O\left(t^{\xi}\right),\left|\lambda_{\langle 2: \xi: d\rangle}(t)\right| \in O\left(t^{\xi+1}\right),\left|\lambda_{\langle 4: \xi: d\rangle}(t)\right| \in O\left(t^{\xi+2}\right), \xi \in\{0, \ldots, d-2\},
$$

just like in the proof of Lemma 16. Considering the prism

$$
\left\{\left(x_{1}, x_{2}, \ldots, x_{d}\right) \mid x_{1}, \ldots, x_{d} \geq 0, x_{1}+x_{2} \leq a, x_{\ell} \leq a \forall_{\ell \in\{3, \ldots, d\}}\right\},
$$

one gets

$$
\left|\lambda_{\langle 1: \xi: d\rangle}(t)+\lambda_{\langle 3: \xi: d\rangle}(t)\right| \in O\left(t^{\xi+1}\right), \quad \xi \in\{0, \ldots, d-2\} .
$$

Since $\left|V_{2}\right|(A) \leq 1,\left|V_{1}\right|(A) \leq 2$ and $\left|V_{0}\right|(A) \leq 1$, where $A$ is the set from the proof of Lemma 16, Corollary 11 yields

$$
\left|V_{k}\right|\left(A \times[-h / 2 ; h / 2]^{d-2}\right) \leq\left(\begin{array}{c}
d-2 \\
k
\end{array}\right) h^{k}+2\left(\begin{array}{l}
d-2 \\
k-1
\end{array}\right) h^{k-1}+\left(\begin{array}{c}
d-2 \\
k-2
\end{array}\right) h^{k-2} \leq\left(\begin{array}{l}
d \\
k
\end{array}\right)
$$

for $h \in(0 ; 1)$ (notice that $\left(\begin{array}{c}d-2 \\ l\end{array}\right) h^{l} \leq\left(\begin{array}{c}d-2 \\ l\end{array}\right)$ holds for all $l \in \mathbb{Z}$; if $l \geq 0$ it holds since $h<1$ and otherwise it holds since $\left.\left(\begin{array}{c}d-2 \\ l\end{array}\right)=0\right)$. The same way we get

$$
\left|V_{k}\right|\left(A_{1} \times[-h / 2 ; h / 2]^{d-2}\right) \leq 2\left(\begin{array}{c}
d-2 \\
k
\end{array}\right) h^{k}+4\left(\begin{array}{l}
d-2 \\
k-1
\end{array}\right) h^{k-1}+\left(\begin{array}{c}
d-2 \\
k-2
\end{array}\right) h^{k-2} \leq 2\left(\begin{array}{l}
d \\
k
\end{array}\right)
$$

for $h \in(0 ; 1)$, where $A_{1}$ is the set from the proof of Lemma 16 .

Hence the asymptotic bias of $\hat{\gamma}$ on $\tilde{A}^{h}:=A \times[-h / 2 ; h / 2]^{d-2}$ and $\tilde{A}_{1}^{h}:=A_{1} \times[-h / 2 ; h / 2]^{d-2}$ may not be arbitrarily large. However, from Theorem 14 we get

$$
\begin{aligned}
\mathbb{E} \hat{\gamma}\left(\tilde{A}_{1}^{h}+t U\right)= & \sum_{\xi=0}^{d-2} \sum_{\iota \in\{1,3,5\}} \lambda_{\langle\iota: \xi: d\rangle}(t) \mathbb{E} \tilde{N}_{t,\langle\iota: \xi: d\rangle}\left(\tilde{A}_{1}^{h}+t U\right) \\
& +\sum_{\iota \in\{2,4\}} \lambda_{\langle\iota: d-2: d\rangle}(t) \mathbb{E} \tilde{N}_{t,\langle\iota: d-2: d\rangle}\left(\tilde{A}_{1}^{h}+t U\right) \\
& +\lambda_{\langle 0: 0: d\rangle}(t) \mathbb{E} \tilde{N}_{t,\langle 0: 0: d\rangle}\left(\tilde{A}_{1}^{h}+t U\right),
\end{aligned}
$$

where $U$ is a random vector distributed uniformly on $[0 ; 1]^{d}$. From (15)-(17) and Corollary 15 we get

$$
\sup _{m_{1}, m_{2}} \limsup _{t \rightarrow 0}\left|\mathbb{E} \hat{\gamma}\left(\tilde{A}_{1}^{h}+t U\right)-\sum_{\xi=0}^{d-2}\left(\begin{array}{c}
d-2 \\
\xi
\end{array}\right) 2^{d-2-\xi}\left(\frac{h}{t}-1\right)^{\xi} \sum_{\iota \in\{1,3,5\}} \lambda_{\langle\iota: \xi: d\rangle}(t) \mathbb{E} \tilde{N}_{t, \iota}\left(A_{1}+t \tilde{U}\right)\right|<\infty,
$$

where $\tilde{U}$ is a random vector distributed uniformly on $[0 ; 1]^{2}$. With $s$ as in the proof of Lemma 16, we conclude just like in that proof

$$
\sup _{m_{1}, m_{2}} \limsup _{t \rightarrow 0}\left|\mathbb{E} \hat{\gamma}\left(\tilde{A}_{1}^{h}+t U\right)-\sum_{\xi=0}^{d-2}\left(\begin{array}{c}
d-2 \\
\xi
\end{array}\right) 2^{d-2-\xi}\left(\frac{h}{t}-1\right)^{\xi}\left(2 \lambda_{\langle 3: \xi: d\rangle}(t)+\lambda_{\langle 5: \xi: d\rangle}(t)\right) \cdot 2 s\right|<\infty .
$$

Hence

$$
\begin{aligned}
o\left(t^{0}\right) & \ni\left|\sum_{\xi=0}^{d-2}\left(\begin{array}{c}
d-2 \\
\xi
\end{array}\right) 2^{d-2-\xi}\left(\frac{h}{t}-1\right)^{\xi}\left(2 \lambda_{\langle 3: \xi: d\rangle}(t)+\lambda_{\langle 5: \xi: d\rangle}(t)\right)\right| \\
& =\left|\sum_{\xi=0}^{d-2} \sum_{k=0}^{\xi}\left(\begin{array}{c}
d-2 \\
\xi
\end{array}\right) 2^{d-2-\xi}\left(\begin{array}{l}
\xi \\
k
\end{array}\right)\left(\frac{h}{t}\right)^{k}(-1)^{\xi-k}\left(2 \lambda_{\langle 3: \xi: d\rangle}(t)+\lambda_{\langle 5: \xi: d\rangle}(t)\right)\right| \\
& =\left|\sum_{k=0}^{d-2}\left(\frac{h}{t}\right)^{k} \sum_{\xi=k}^{d-2}\left(\begin{array}{c}
d-2 \\
\xi
\end{array}\right) 2^{d-2-\xi}\left(\begin{array}{l}
\xi \\
k
\end{array}\right)(-1)^{\xi-k}\left(2 \lambda_{\langle 3: \xi: d\rangle}(t)+\lambda_{\langle 5: \xi: d\rangle}(t)\right)\right| .
\end{aligned}
$$




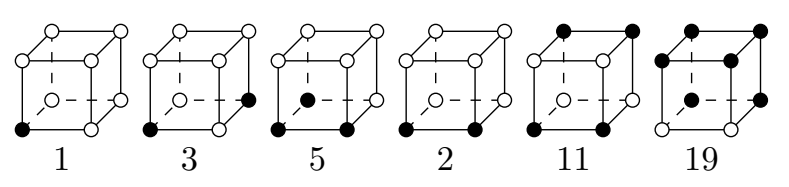

Figure 3: Representatives of the equivalence classes 1, 3, 5, 2, 11 and 19

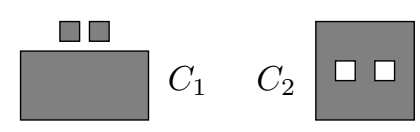

Figure 4: Idea of the construction of $C_{1}$ and $C_{2}$ for $n=3$ : Both sets have the same area and the same perimeter.

Since $h$ was arbitrary, we get

$$
\left|\frac{1}{t^{k}} \sum_{\xi=k}^{d-2}\left(\begin{array}{c}
d-2 \\
\xi
\end{array}\right) 2^{d-2-\xi}\left(\begin{array}{l}
\xi \\
k
\end{array}\right)(-1)^{\xi-k}\left(2 \lambda_{\langle 3: \xi: d\rangle}(t)+\lambda_{\langle 5: \xi: d\rangle}(t)\right)\right| \in o\left(t^{0}\right), \quad k \in\{0, \ldots, d-2\},
$$

which yields $\left|2 \lambda_{\langle 3: \xi: d\rangle}(t)+\lambda_{\langle 5: \xi: d\rangle}(t)\right| \in o\left(t^{\xi}\right)$. The same way one gets

$$
\sup _{m_{1}, m_{2}} \limsup _{t \rightarrow 0}\left|\mathbb{E} \hat{\gamma}\left(\tilde{A}^{h}+t U\right)-\sum_{\xi=0}^{d-2}\left(\begin{array}{c}
d-2 \\
\xi
\end{array}\right) 2^{d-2-\xi}\left(\frac{h}{t}-1\right)^{\xi}\left(2 \lambda_{\langle 1: \xi: d\rangle}(t)+\lambda_{\langle 5: \xi: d\rangle}(t)\right) \cdot 2 s\right|<\infty
$$

and thus $\left|2 \lambda_{\langle 1: \xi: d\rangle}(t)+\lambda_{\langle 5: \xi: d\rangle}(t)\right| \in o\left(t^{\xi}\right)$. Now Lemma 17 implies $\left|\lambda_{\langle 1: \xi: d\rangle}(t)\right|,\left|\lambda_{\langle 3: \xi: d\rangle}(t)\right|,\left|\lambda_{\langle 5: \xi: d\rangle}(t)\right| \in$ $o\left(t^{\xi}\right)$.

Example 19. In the special case $d=3$ Lemma 18 yields

$$
\begin{aligned}
\left|\lambda_{1}(t)\right|,\left|\lambda_{3}(t)\right|,\left|\lambda_{5}(t)\right| & \in o\left(t^{0}\right) \\
\left|\lambda_{2}(t)\right|,\left|\lambda_{11}(t)\right|,\left|\lambda_{19}(t)\right| & \in o(t)
\end{aligned}
$$

where we use the enumeration of the equivalence classes from [7]. Representatives of the equivalence classes 1, 3, 5, 2, 11 and 19 are shown in Figure 3.

Proof of Theorem 1. We start by proving the theorem in the special case $d=2$.

Consider an arbitrary estimator $\hat{V}_{0}$ of type (1). Let $R_{1} \subseteq(-1 ; 1)^{2}$ be an axes-parallel rectangle with sidelengths 0.9 and 1.1 and put $C_{1}:=R_{1} \cup \bigcup_{i=1}^{n-1}\left(\lambda[0 ; 1]^{2}+v_{i}\right)$, where $\lambda:=\sqrt{\frac{1}{100 \cdot(2 n-2)}}$ and $v_{1}, \ldots, v_{n-1} \in \mathbb{R}^{2}$ are vectors such that the union of all $n$ sets is disjoint and contained in $(-1 ; 1)^{2}$. Let $R_{2}$ be an axes-parallel square of side-length 1 and set $C_{2}:=R_{2} \backslash \bigcup_{i=1}^{n-1} \operatorname{int}\left(\lambda[0 ; 1]^{2}+w_{i}\right)$, where $w_{1}, \ldots, w_{n-1}$ are vectors such that $\lambda[0 ; 1]^{2}+w_{1}, \ldots, \lambda[0 ; 1]^{2}+w_{n-1}$ are pairwise disjoint subsets of int $R_{2}$ (see Figure 4 ). By Lemma 7 we have $\left|V_{0}\right|\left(C_{1}\right) \leq n$ and $\left|V_{0}\right|\left(C_{2}\right) \leq n$.

Let $U$ be a random vector distributed uniformly on $[0 ; 1]^{2}$ and let $t \in(0 ; \lambda)$ be smaller than the smallest distance between two connected components of $C_{1}$ or $(-1 ; 1)^{2} \backslash C_{2}$ divided by $\sqrt{2}$ and also smaller than half the distance between bd $[-1 ; 1]^{2}$ and $C_{1}$ or $C_{2}$. Noticing

$$
V_{2}\left(C_{1}\right)=0.9 \cdot 1.1+\frac{1}{200}=0.995=1-\frac{1}{200}=V_{2}\left(C_{2}\right),
$$


we get

$$
\begin{array}{ll}
\mathbb{E} \tilde{N}_{t, 1}\left(C_{1}+t U\right)=4 n, & \mathbb{E} \tilde{N}_{t, 1}\left(C_{2}+t U\right)=4, \\
\mathbb{E} \tilde{N}_{t, 2}\left(C_{1}+t U\right)=4\left(\frac{1}{t}-1\right)+4(n-1)\left(\frac{\lambda}{t}-1\right), & \mathbb{E} \tilde{N}_{t, 2}\left(C_{2}+t U\right)=4\left(\frac{1}{t}-1\right)+4(n-1)\left(\frac{\lambda}{t}-1\right), \\
\mathbb{E} \tilde{N}_{t, 3}\left(C_{1}+t U\right)=0, & \mathbb{E} \tilde{N}_{t, 3}\left(C_{2}+t U\right)=4 n-4, \\
\mathbb{E} \tilde{N}_{t, 4}\left(C_{1}+t U\right)=\frac{0.995}{t^{2}}-\frac{2+2(n-1) \lambda}{t}+n, & \mathbb{E} \tilde{N}_{t, 4}\left(C_{2}+t U\right)=\frac{0.995}{t^{2}}-\frac{2+2(n-1) \lambda}{t}+2-n, \\
\mathbb{E} \tilde{N}_{t, 5}\left(C_{1}+t U\right)=0, & \mathbb{E} \tilde{N}_{t, 5}\left(C_{2}+t U\right)=0, \\
\mathbb{E} \tilde{N}_{t, 0}\left(C_{1}+t U\right)=r(t)-\frac{0.995}{t^{2}}-\frac{2+2(n-1) \lambda}{t}-n, & \mathbb{E} \tilde{N}_{t, 0}\left(C_{2}+t U\right)=r(t)-\frac{0.995}{t^{2}}-\frac{2+2(n-1) \lambda}{t}+n-2,
\end{array}
$$

where $r(t)$ was defined in the proof of Lemma 16 .

Now Lemma 16 yields

$$
\begin{aligned}
\limsup _{t \rightarrow 0} \mid \mathbb{E} & {\left[\hat{V}_{0}\left(C_{1}+t U\right)-\hat{V}_{0}\left(C_{2}+t U\right)\right] \mid } \\
& =\limsup _{t \rightarrow 0}\left|(4 n-4) \cdot \lambda_{1}(t)+(4-4 n) \cdot \lambda_{3}(t)+(2 n-2) \cdot \lambda_{4}(t)+(2-2 n) \cdot \lambda_{0}(t)\right| \\
& =0
\end{aligned}
$$

if the worst case asymptotic bias of $\hat{V}_{0}$ is finite. Since $V_{0}\left(C_{1}\right)=n$ and $V_{0}\left(C_{2}\right)=2-n$, this yields either $\lim \sup _{t \rightarrow 0}\left|\mathbb{E} \hat{V}_{0}\left(C_{1}+t U\right)-V_{0}\left(C_{1}\right)\right| \geq n-1$ or $\limsup _{t \rightarrow 0}\left|\mathbb{E} \hat{V}_{0}\left(C_{2}+t U\right)-V_{0}\left(C_{2}\right)\right| \geq n-1$. Thus, in any case, the worst case asymptotic bias of $\hat{V}_{0}$ is at least $n-1$.

On the other hand, consider the estimator that always returns 1 . The positive part of $\Xi_{0}(K, \cdot)$ is at least 1 for any set $K \subseteq \mathbb{R}^{2}$ from the convex ring by Lemma 5 . Hence for all sets $K$ with $\left|V_{0}\right|(K) \leq n$ we have $2-n \leq V_{0}(K) \leq n$. So the estimator that always returns 1 has a worst case (asymptotic) bias of $n-1$.

Now we turn to the case $d>2$.

Let $C_{1} \subseteq \mathbb{R}^{2}$ and $C_{2} \subseteq \mathbb{R}^{2}$ be the sets defined above and put $D_{\nu}:=C_{\nu} \times\left[-\frac{1}{2} ; \frac{1}{2}\right]^{d-2}, \nu=1,2$. Then $D_{1}, D_{2} \subseteq(-1 ; 1)^{d}$ have both a total variation of the 0 -th support measure of at most $n$ and $V_{0}\left(D_{1}\right)=n$ and $V_{0}\left(D_{2}\right)=2-n$ by Corollary 10 .

For sufficiently small $t>0$ Theorem 15 yields

$$
\mathbb{E} \tilde{N}_{t,\langle 3: \xi: d\rangle}\left(D_{1}+t U\right)=0, \quad \mathbb{E} \tilde{N}_{t,\langle 3: \xi: d\rangle}\left(D_{2}+t U\right)=\left(\begin{array}{c}
d-2 \\
\xi
\end{array}\right) 2^{d-2-\xi}\left(\frac{1}{t}-1\right)^{\xi} \cdot(4 n-4)
$$

and

$$
\begin{aligned}
\mathbb{E} \tilde{N}_{t,\langle 1: \xi: d\rangle} & \left(D_{1}+t U\right)-\mathbb{E} \tilde{N}_{t,\langle 1: \xi: d\rangle}\left(D_{2}+t U\right) \\
& =\left(\begin{array}{c}
d-2 \\
\xi
\end{array}\right) 2^{d-2-\xi}\left(\frac{1}{t}-1\right)^{\xi} \cdot\left[\mathbb{E} \tilde{N}_{t, 1}\left(C_{1}+t \tilde{U}\right)-\mathbb{E} \tilde{N}_{t, 1}\left(C_{2}+t \tilde{U}\right)\right] \\
& +\left(\begin{array}{c}
d-2 \\
\xi-1
\end{array}\right) 2^{d-1-\xi}\left(\frac{1}{t}-1\right)^{\xi-1} \cdot\left[\mathbb{E} \tilde{N}_{t, 2}\left(C_{1}+t \tilde{U}\right)-\mathbb{E} \tilde{N}_{t, 2}\left(C_{2}+t \tilde{U}\right)\right] \\
& +\left(\begin{array}{c}
d-2 \\
\xi-2
\end{array}\right) 2^{d-\xi}\left(\frac{1}{t}-1\right)^{\xi-2} \cdot\left[\mathbb{E} \tilde{N}_{t, 4}\left(C_{1}+t \tilde{U}\right)-\mathbb{E} \tilde{N}_{t, 4}\left(C_{2}+t \tilde{U}\right)\right] \\
& =\left(\begin{array}{c}
d-2 \\
\xi
\end{array}\right) 2^{d-2-\xi}\left(\frac{1}{t}-1\right)^{\xi} \cdot(4 n-4)+\left(\begin{array}{c}
d-2 \\
\xi-2
\end{array}\right) 2^{d-\xi}\left(\frac{1}{t}-1\right)^{\xi-2} \cdot(2 n-2)
\end{aligned}
$$

for $\xi \in\{0, \ldots, d-2\}$, where $U$ is a random vector distributed uniformly on $[0 ; 1]^{d}$ and $\tilde{U}$ is a random vector distributed uniformly on $[0 ; 1]^{2}$. Moreover,

$$
\mathbb{E} \tilde{N}_{t,\langle 2: d-2: d\rangle}\left(D_{1}+t U\right)-\mathbb{E} \tilde{N}_{t,\langle 2: d-2: d\rangle}\left(D_{2}+t U\right)=(d-2) 2\left(\frac{1}{t}-1\right)^{d-3} \cdot(2 n-2)
$$

and

$$
\mathbb{E} \tilde{N}_{t,\langle 4: d-2: d\rangle}\left(D_{1}+t U\right)-\mathbb{E} \tilde{N}_{t,\langle 4: d-2: d\rangle}\left(D_{2}+t U\right)=\left(\frac{1}{t}-1\right)^{d-2} \cdot(2 n-2) .
$$


The equality

$$
\mathbb{E} \tilde{N}_{t, j}\left(D_{1}+t U\right)=\mathbb{E} \tilde{N}_{t, j}\left(D_{2}+t U\right)=0
$$

follows from Theorem 15 for all equivalence classes $j$ of type $\langle 5: \xi: d\rangle, \xi \in\{0, \ldots, d-2\}$, and from Corollary 14 for all remaining equivalence classes except $\langle 0: 0: d\rangle$. Thus Lemma 18 implies

$$
\begin{aligned}
& \limsup _{t \rightarrow 0}\left|\mathbb{E}\left[\hat{V}_{0}\left(D_{1}+t U\right)-\hat{V}_{0}\left(D_{2}+t U\right)\right]\right| \leq \\
& \limsup _{t \rightarrow 0}\left(\sum_{\xi=0}^{d-2}\left(\left(\begin{array}{c}
d-2 \\
\xi
\end{array}\right) 2^{d-2-\xi}\left(\frac{1}{t}-1\right)^{\xi} \cdot(4 n-4)+\left(\begin{array}{c}
d-2 \\
\xi-2
\end{array}\right) 2^{d-\xi}\left(\frac{1}{t}-1\right)^{\xi-2} \cdot(2 n-2)\right) \cdot\left|\lambda_{\langle 1: \xi: d\rangle}(t)\right|\right. \\
& \quad+\sum_{\xi=0}^{d-2}\left(\begin{array}{c}
d-2 \\
\xi
\end{array}\right) 2^{d-2-\xi}\left(\frac{1}{t}-1\right)^{\xi} \cdot(4 n-4) \cdot\left|\lambda_{\langle 3: \xi: d\rangle}(t)\right| \\
& \quad+(d-2) 2\left(\frac{1}{t}-1\right)^{d-3} \cdot(2 n-2) \cdot\left|\lambda_{\langle 2: d-2: d\rangle}(t)\right| \\
&\left.\quad+\left(\frac{1}{t}-1\right)^{d-2} \cdot(2 n-2) \cdot\left|\lambda_{\langle 4: d-2: d\rangle}(t)\right|\right) \\
&=0
\end{aligned}
$$

if the worst case asymptotic bias of $\hat{V}_{0}$ is finite. Hence the worst case asymptotic bias of $\hat{V}_{0}$ is at least $n-1$ in any case.

The assertion concerning the constant estimator follows the same way as in the special case $d=2$.

Proof of Theorem 3. Let $\epsilon \in(0 ; 1)$. Set $D_{\nu}:=C_{\nu} \times[-1 / 2 ; 1 / 2]^{d-k-2}, \nu=1,2$, where $C_{1}$ and $C_{2}$ are the sets from the proof of Theorem 1 for some $n \geq 6 r / \epsilon$. Put

$$
\begin{aligned}
\rho & :=\sqrt[k]{\frac{r-\epsilon / 3}{n}}, \\
\mu & :=\frac{\epsilon}{3 \max \left(\left\{\sum_{i=1}^{\min \{k, d-k\}}\left(\begin{array}{c}
k \\
i
\end{array}\right) \rho^{k-i}\left|V_{i}\right|\left(D_{\nu}\right) \mid \nu=1,2\right\} \cup\{\epsilon\}\right)},
\end{aligned}
$$

and $E_{\nu}:=\mu C_{\nu} \times[-\mu / 2 ; \mu / 2]^{d-k-2} \times[-\rho / 2 ; \rho / 2]^{k}, \nu=1,2$. Since we have shown $V_{0}\left(D_{1}\right)=n$ and $V_{0}\left(D_{2}\right)=2-n$ in the proof of Theorem 1 , Corollary 11 gives

$$
\begin{aligned}
V_{k}\left(E_{1}\right) & =\sum_{i=0}^{\min \{k, d-k\}}\left(\begin{array}{c}
k \\
i
\end{array}\right) \rho^{k-i} \mu^{i} V_{i}\left(D_{1}\right) \\
& \geq \rho^{k} V_{0}\left(D_{1}\right)-\mu \cdot \sum_{i=1}^{\min \{k, d-k\}}\left(\begin{array}{c}
k \\
i
\end{array}\right) \rho^{k-i}\left|V_{i}\right|\left(D_{1}\right) \\
& \geq \frac{r-\epsilon / 3}{n} \cdot n-\frac{\epsilon}{3} \\
& >r-\epsilon
\end{aligned}
$$

and

$V_{k}\left(E_{2}\right) \leq \rho^{k} V_{0}\left(D_{2}\right)+\mu \cdot \sum_{i=1}^{\substack{\min \{k, d-k\}}}\left(\begin{array}{c}k \\ i\end{array}\right) \rho^{k-i}\left|V_{i}\right|\left(D_{2}\right) \leq \frac{r-\epsilon / 3}{n} \cdot(2-n)+\frac{\epsilon}{3}=\frac{2 r}{n}-r-\frac{2 \epsilon}{3 n}+\frac{\epsilon}{3}+\frac{\epsilon}{3} \leq-r+\epsilon$.

Similar as above, we get for $\nu=1,2$

$$
\left|V_{k}\right|\left(E_{\nu}\right) \leq \sum_{i=0}^{\min \{k, d-k\}}\left(\begin{array}{l}
k \\
i
\end{array}\right) \rho^{k-i} \mu^{i}\left|V_{i}\right|\left(D_{\nu}\right) \leq \rho^{k}\left|V_{0}\right|\left(D_{\nu}\right)+\mu \cdot \sum_{i=1}^{\min \{k, d-k\}}\left(\begin{array}{c}
k \\
i
\end{array}\right) \rho^{k-i}\left|V_{i}\right|\left(D_{\nu}\right) \leq r .
$$


Moreover, we get

$$
\limsup _{t \rightarrow 0}\left|\mathbb{E}\left[\hat{V}_{k}\left(E_{1}+t U\right)-\hat{V}_{k}\left(E_{2}+t U\right)\right]\right|=0
$$

the same way as $(21)$, where $U$ is a random vector distributed uniformly on $[0 ; 1]^{d}$. Hence we obtain $\lim \sup _{t \rightarrow 0}\left|\mathbb{E} \hat{V}_{k}\left(E_{1}+t U\right)-V_{k}\left(E_{1}\right)\right| \geq r-\epsilon$ or $\limsup _{t \rightarrow 0}\left|\mathbb{E} \hat{V}_{k}\left(E_{2}+t U\right)-V_{k}\left(E_{2}\right)\right| \geq r-\epsilon$. Since $\epsilon>0$ was arbitrary, the worst case bias of $\hat{V}_{k}$ is at least $r$.

\section{References}

[1] S.B. Gray: Local properties of binary images in two dimensions, IEEE Transactions on Computers C-20 (1971) 551-561.

[2] M. Kiderlen: Estimating the Euler characteristic of a planar set from a digital image, Journal of Visual Communication and Image Representation 17 (2006) 1237-1255.

[3] M. Kiderlen, J. Rataj: On infinitesimal increase of volumes of morphological transforms, Mathematika 53 (2006) 103-127.

[4] M. Kiderlen, J. Ziegel: Estimation of surface area and surface area measure of three-dimensional sets from digitizations, Image Vision and Computing 28 (2010) 64-77.

[5] J. Lindblad: Surface area estimation of digitized 3D objects using weighted local configurations, Image and Vision Computing 23 (2005) 111-122.

[6] D. Meschenmoser, E. Spodarev: On the computation of intrinsic volumes, Preprint.

[7] J. Ohser, W. Nagel, K. Schladitz: Miles formulae for Boolean models observed on lattices, Image Analysis and Stereology 28 (2009), 77-92.

[8] J. Ohser, K. Sandau, J. Kampf, I. Vecchio, A. Moghiseh: Improved estimation of fibre length from 3-dimensional images, Preprint.

[9] R. Schneider: Convex Bodies: The Brunn-Minkowski Theory, Cambrigde University Press, 1993.

[10] R. Schneider, W. Weil: Stochastic and Integral Geometry, Springer, Berlin, Heidelberg, 2008. 